\title{
Marker Network for Bladder Cancer
}

National Cancer Institute

\section{Source}

National Cancer Institute. Marker Network for Bladder Cancer. NCI Thesaurus. Code C16073.

The goal of the network is to evaluate biochemical, immunologic, genetic, and other quantifiable markers for diagnosis and prognosis of urinary bladder cancer. 